\title{
Migración de afiliados en el Sistema Isapre
}

\author{
MANUEL INOSTROZA(1), ALBERTO MUÑOZ(2) y MARLENE SÁNCHEZ(2)
}

\section{INTRODUCCIÓN}

Desde 1998, el Sistema Isapre viene experimentando una pérdida sistemática de beneficiarios. Aunque esta disminución de cartera muestra una tendencia anual decreciente, la pérdida neta total de beneficiarios asciende a 1.224.202, a contar de diciembre de 1997, período durante el cual el Sistema alcanzó un máximo de 3.882 .572 personas. Sólo en lo que va corrido de 2005 , la pérdida de cartera alcanza aproximadamente a los 20.637 beneficiarios, lo que indica que la migración de afiliados sigue siendo un tema para esta industria.

Variadas son las causas que explican la migración, entre ellas se distinguen: la crisis asiática de finales de los noventa y su impacto negativo sobre las tasas de empleo y poder adquisitivo de las personas; el mejoramiento continuo de los servicios de atención del Fonasa, percibido favorablemente por la población de isapre; $y$, la reciente incorporación del Plan Auge (GES) que no ha sido promocionado adecuadamente por las isapres, con el consiguiente fortalecimiento de la competitividad del seguro público. Otras causas se atribuyen a las fallas estructurales del Sistema, que posibilitaban la adecuación anual tanto de precios como de beneficios junto a la desafiliación voluntaria por parte de los afiliados, condiciones que transformaron al contrato de salud privado en un seguro de corto plazo que no tomaba en consideración el riesgo ni las necesidades de salud futuras de los usuarios, aspectos muchos de los cuales, fueron recogidos y mejorados con la Reforma. Finalmente, otras causas son imputables a las propias isapres y a su falta de iniciativas orientadas a fidelizar a los clientes y a promover su permanencia; por ejemplo, a través de la implementación de programas de tipo preventivo o de mayor protección para la tercera edad o mediante el traspaso de sus mayores utilidades a nuevos beneficios y/o menores precios para sus beneficiarios, incentivos que, de ahora en adelante, les impone la propia Reforma, con una mayor estandarización de planes de salud, regulación de precios y modelos de atención que enfatizan la prevención y promoción, médicos de familia, compra de soluciones integrales en salud, entre otras.

Entre 1997 y 1998 el número de desahucios de contratos pasa a ser superior al número de suscripciones ocurridas en cada período (Figura 1), determinando una tasa de variación negativa a partir de ese punto de inflexión, que muestra una tendencia decreciente con aproximación al equilibrio en los últimos años.

No obstante lo anterior, durante los meses de julio y agosto de 2005 , la tasa de desafiliaciones ha experimentado un nuevo y fuerte incremento como resultado de la puesta en marcha del Plan Auge en el Sistema Isapre, el cual ha determinado un costo adicional para los afiliados. Este incremento de precios, aunque marginal, ha sido percibido negativamente por los beneficiarios, quienes han ejercido su derecho a desahuciar sus contratos con las isapres.

La mayor tasa de desafiliaciones, en este período, implicó la pérdida de 9.809 contratos

(1) Superintendente de Salud.

(2) Departamento de Estudios y Desarrollo, Superintendencia de Salud. 


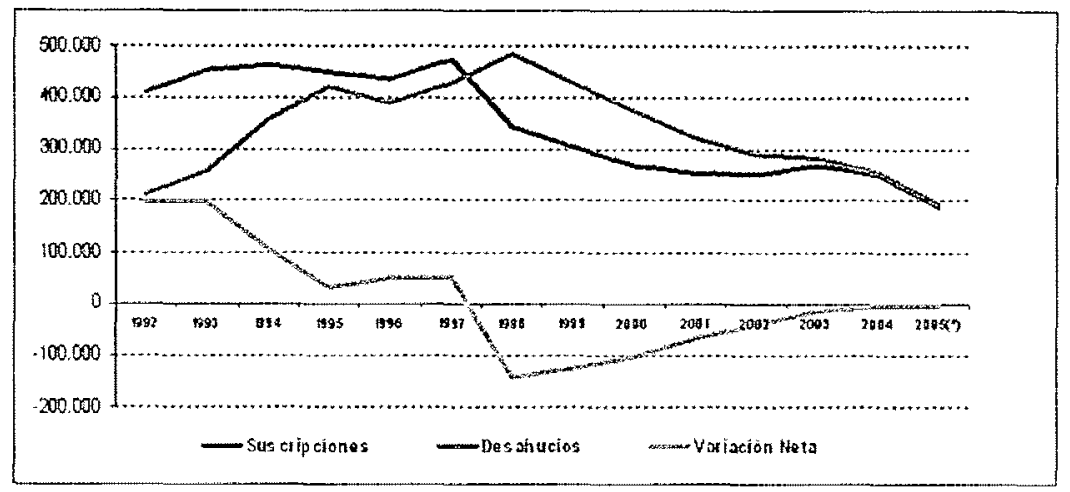

Figura 1. Evolución de las suscripciones y desahucios de contratos.

Elaboración propia sobre la base de información disponible en la Superintendencia de Salud.

de salud y de 22.658 beneficiarios* (Tabla 1 ). Las isapres que se han visto más afectadas son: Consalud, Banmédica, Vida Tres y Normédica.

Del total de desahucios entre agosto de 2004 y agosto de 2005 , un $64,8 \%$ corresponde a desafiliaciones voluntarias de los cotizantes, lo que constituye la principal causa; un $20,3 \%$ a finiquitos cursados por las Isapres y un 14,9\% corresponden a términos por otras causas entre las que se encuentran el mutuo acuerdo de las partes (Tabla 2).

\section{Cotizantes según antigüedad en la Isapre}

Los cotizantes que se quedaron en el Sistema Isapre luego de la crisis asiática, muestran un comportamiento estable, por cuanto, más de

Tabla 1. Suscripciones y desahucios de contratos

\begin{tabular}{lccc}
\hline Período & suscripciones & Desahucios & Variación neta \\
\hline Ene-05 & 19.115 & 17.070 & 2.045 \\
Feb-05 & 14.925 & 15.012 & -87 \\
Mar-05 & 23.378 & 22.511 & 867 \\
Abr-05 & 23.072 & 23.164 & -92 \\
May-05 & 21.761 & 21.964 & -203 \\
Jun-05 & 26.153 & 23.819 & 2.334 \\
Jul-05 & 31.377 & 38.837 & $-7-460$ \\
Ago-05 & 29.433 & 31.782 & -2.349 \\
\hline
\end{tabular}

Tabla 2. Distribución de los desahucios de contratos según causal. Agosto 2004 a agosto de 2005

\begin{tabular}{lcccc}
\hline & Voluntarios & $\begin{array}{c}\text { Desahucios } \\
\text { Parte Isapre }\end{array}$ & Otra causal & Total desahucios \\
\hline Número de contratos & 191.274 & 60.084 & 43.936 & 295.294 \\
Distribución porcentual & $64,8 \%$ & $20,3 \%$ & $14,9 \%$ & $100,0 \%$ \\
\hline
\end{tabular}

Fuente: Superintendencia de Salud.

Proyecciones efectuadas sobre la base de las suscripciones y desaliucios de contratos ocurridos en julio y agosto de 2005 , considerando el número de cargas promedio por cotizante en cada isapre. 


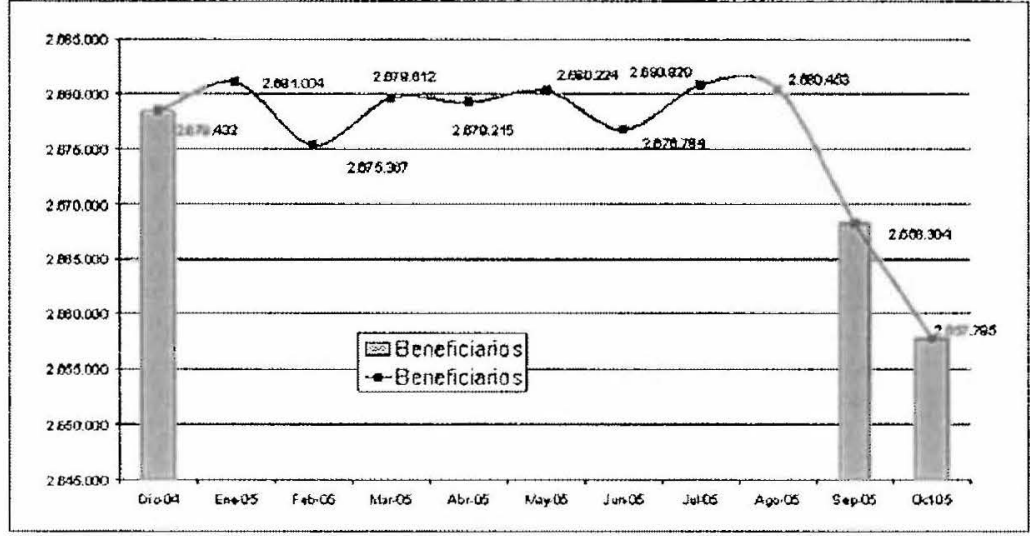

Figura 2. Evolución de los beneficiarios Año 2005.

Elaboración propia sobre la base de información disponible en la Superintendencia de Salud.

un $70 \%$ han permanecido fieles a su Isapre por más de 3 años y un $25,3 \%$ lo ha hecho por más de 10 años (Figura 3).

Al distribuir la cartera de cotizantes antiguos de agosto de $2005^{* *}$, por tramos de permanencia en sus respectivos contratos de salud, se puede observar que un $9,2 \%$ de los cotizantes presenta una antigüedad inferior a un año; un $19,9 \%$, entre 1 y 3 años; un $26,3 \%$ entre 3 y 6 años; un $19,3 \%$ entre 6 y 10 años; y un $25,3 \%$ presenta una antigüedad de más de 10 años en sus respectivos contratos y por ende, en la misma isapre.

Al efectuar el análisis por isapre, se observa, que las isapres cerradas, como es natural, presentan un mayor nivel de fidelidad por parte de sus cotizantes. Las isapres abiertas en cambio, concentran su cartera de cotizantes en el tramo de antigüedad que corresponde a 3 y 6 años de permanencia (26,2\%). Las isapres abiertas que destacan por una mayor proporción de cotizantes fieles, son: Consalud, con un $32,6 \%$ de su cartera en el tramo de antigüedad superior a 10 años; Banmédica, con un 29,2\%; Colmena, con un 22,6\% y; Normédica con un $22,6 \%$. Por otra parte, las isapres abiertas que se destacan por presentar una mayor proporción de cotizantes en tramo de antigüedad inferior a un año, son: Sfera, con un $13,6 \%$ de su cartera en este tramo y Masvida, con un $10,4 \%$.

\section{CARACTERIZACIÓN DE LOS COTIZANTES SEGÚN PROPIEDADES DE MIGRACIÓN}

Cotizantes que permanecieron en el Sistema

Un $89 \%$ de los cotizantes de agosto de 2004 permanecieron en el Sistema Isapre, al menos, hasta agosto de 2005. De ellos, un $87,7 \%$ continuaron en la misma isapre mientras el $12,3 \%$ restante se cambio de institución $^{* * * *}$.

Al comparar las características de los cotizantes que permanecieron en el Sistema Isapre entre agosto 2004 y agosto 2005, se observa que: la cotización pactada promedio aumentó en un $6 \%$ real, la cotización adicional promedio aumentó en un $10,9 \%$ real, la edad promedio aumentó en un año, tal como se podía esperar y el número de cargas promedio aumentó en 0,01 .

La principal diferencia entre los cotizantes que se cambiaron de isapre y aquéllos que continuaron en ella, radica en que los primeros tienen un menor poder adquisitivo, un menor

** La cartera de cotizantes antiguos de agosto de 2005. no considera los cotizantes que se incorporaron al Sistema 1sapre, por primera vez, en los últimos tres años. Esta cartera representa un $79,1 \%$ del total de cotizantes del período.

*** Para determina la población de cotizantes que permaneció en el Sistema lsapre. se utilizó la información correspondiente a los Archivos Maestros de Beneficiarios de agosto 2004 (período inicial) y agosto 2005 (periodo final). De esta forma, los cotizantes que permanecieron en el Sistema, se definen como aquéllos cuyos RUT aparecen en ambos periodos. Este cruce entrega información para el $95,5 \%$ de la cartera. 


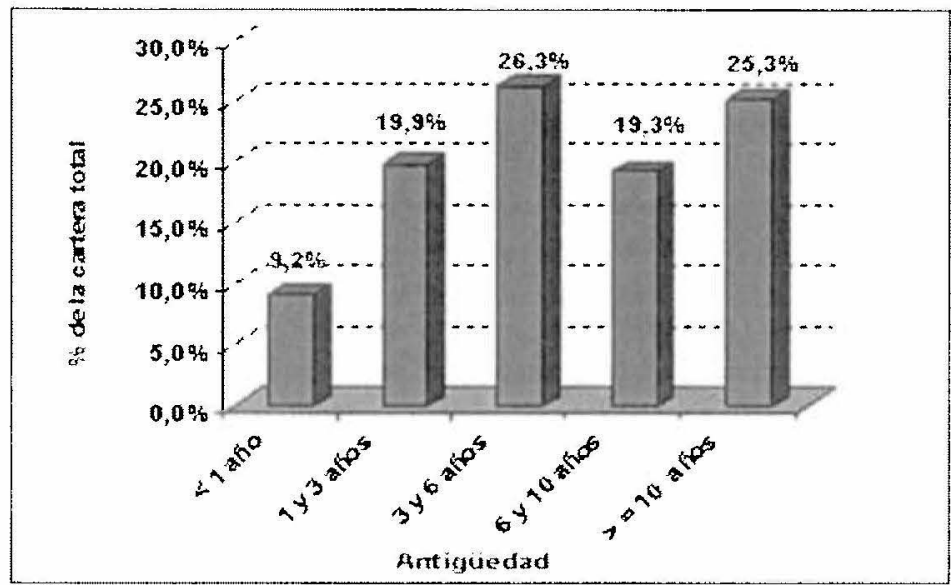

Figura 3. Cotizantes según antigüedad en la isapre.

Elaboración propia a partir de información disponible en la Superintendencia de Salud.

Tabla 3. Características de la cartera de cotizantes que abandonó el sistema.

(Valores a agosto de 2004)

\begin{tabular}{lcccccc}
\hline & $\begin{array}{c}\mathbf{N}^{*} \\
\text { Cotizantes }\end{array}$ & $\begin{array}{c}\text { Cotización } \\
\text { pactada }\end{array}$ & $\begin{array}{c}\text { Cotización } \\
\text { adicional }\end{array}$ & $\begin{array}{c}\text { Número } \\
\text { cargas }\end{array}$ & $\begin{array}{c}\text { Edad } \\
\text { cotizante }\end{array}$ & Masculino \\
\hline Permanecen (2004) & 1.089 .452 & 56.660 & 14.334 & 1,23 & 41,7 & $65,00 \%$ \\
Abandonan (2004) & 129.314 & 36.613 & 6.610 & 0,88 & 36,9 & $68,20 \%$ \\
\hline
\end{tabular}

número de cargas y presentan, en promedio, 5 años menos de edad.

\section{Cotizantes que abandonaron el Sistema}

Un $11 \%$ de la cartera de cotizantes de agosto de 2004, abandonó el Sistema Isapre entre septiembre de 2004 y agosto de $2005^{* * * *}$.

Al comparar las características de los cotizantes que permanecieron en el Sistema con las características de los cotizantes que lo abandonaron, se observa que: la cotización pactada promedio de los que abandonaron, es menor a la de los que permanecieron, en $\$ 20.047$; la cotización adicional promedio de los que abandonaron, es menor a la de los que permanecieron, en $\$ 7.724$; la edad promedio de los que abandonaron, es menor a la de los que permanecieron, en 4,8 años; el número de cargas promedio de los que abandonaron, es menor a la de los que permanecieron, en 0,35 cargas y que la proporción de hombres que abandonaron es mayor a la de los que permanecieron, en 3 puntos porcentuales ${ }^{* * * * * *}$ (Tabla 3).

El análisis realizado permite concluir que los cotizantes que abandonaron el Sistema Isapre presentan un menor poder adquisitivo, son más jóvenes y tienen un menor número de cargas que aquéllos que permanecieron en el Sistema.

\section{COMENTARIOS}

El Sistema Isapre ha experimentado desde 1998 una sustancial pérdida de beneficiarios, aproximadamente un $32 \%$ de su cartera. Han transcurrido 8 años desde entonces y la migración de beneficiarios sigue siendo un

**** Para determinar la población de cotizantes que abandonó el Sistema lsapre, se ulilizó la información correspondiente a los Archivos Maestros de Beneficiarios de agosto 2004 (periodo inicial) y agosto 2005 (período final). De esta forma, los cotizantes que abandonaron el Sistema, se definen como aquéllos cuyos RUT aparecen en el período inicial y no aparecen en el período final. Este cruce entrega información para el 95,5\% de la cartera.

***** La comparación considera la información de los subgrupos a agosto de 2004 , ya que, en agoslo de 2005 , no existe informacion referida a los cotizantes que abandonaron el Sistema. 
problema por resolver en esta industria.

La principal causa de los desahucios, es la desafiliación voluntaria, la cual explica cerca del $65 \%$ de los casos, mostrando el grado de sensibilidad que los cotizantes presentan frente a la calidad de los servicios ofrecidos y al precio de los planes de salud. Hasta ahora, los cotizantes que han abandonado el Sistema presentan, en promedio, un menor poder adquisitivo, menor edad y un menor número de cargas.

Aunque los cotizantes que permanecieron en el Sistema se muestran mayoritariamente estables, persiste entre ellos el riesgo de abandono mientras los planes de salud continúen encareciéndose. Se debe tener presente que la cotización pactada de estos cotizantes ha experimentado un incremento promedio de $6 \%$ real, sólo en el último año.

Lo anterior, denota una actitud pasiva de parte de las isapres para fidelizar a sus clientes y una clara falta de iniciativa para generar mecanismos que permitan incentivar la permanencia de los beneficiarios en el Sistema. Esto, por cuanto las utilidades obtenidas en los últimos 3 años no se han visto reflejadas en mayores y mejores beneficios para los usuarios, y mucho menos en una rebaja de los precios cobrados por los planes de salud. Sólo en el primer semestre de 2005, el Sistema presenta una rentabilidad promedio de $8,7 \%$ sobre el ingreso operacional, mostrando un $28,5 \%$ de incremento en las utilidades respecto de igual período del año anterior. Estos resultados proporcionan un respaldo financiero a las isapres para enfrentar la competencia del Fonasa.

Se debe tener presente que en los últimos años, el mejoramiento continuo de la atención ofrecida por el Fonasa, como así también, las prácticas comerciales asumidas por las isapres junto a la reciente incorporación del Plan Auge, han fortalecido al sector público de la salud, haciéndolo más competitivo frente a las isapres, generando con ello una nueva amenaza para esta industria.

En definitiva, las isapres deberán asumir tanto las nuevas posibilidades como los desafíos que les establece la Reforma. En otras palabras, cambiar el modelo de atención y la forma de resolver los problemas de salud de su cartera, con un mayor énfasis en lo preventivo y promocional, en la conformación de redes de atención con una mejor mezcla entre lo ambulatorio y lo hospitalario y en soluciones más integrales y eficientes de los problemas de salud de la población en todo su ciclo de vida, incluso con la incorporación, entre otros, de medicamentos ambulatorios. De no considerar los incentivos que la Reforma les impone, las isapres continuarán disminuyendo su participación e importancia en el Sistema de Salud.

Usted puede comentar éste y otros artículos publicados en la Revista Chilena de Salud Pública, enviando un correo electrónico a revistasp@med.uchile.cl 